\title{
Effect of Selenomethionine on Growth of Escherichia coli and Bacillus megaterium
}

\author{
M. WU AND J. T. WACHSMAN ${ }^{2}$ \\ Department of Microbiology, University of Illinois, Urbana, Illinois 61801
}

Received for publication 8 September 1970

\begin{abstract}
Studies with methionineless strains of Escherichia coli WWU and Bacillus megaterium KM have shown that selenomethionine only partially satisfies their methionine requirement.
\end{abstract}

This study was based upon the report (2) that selenomethionine (Se-M) could completely replace methionine (S-M) for the normal exponential growth of Escherichia coli ML-30. The growth studies reported here were preliminary to experiments designed to determine whether the methyl group of Se-M could serve as an effective methyl donor for nucleic acids.

Bacillus megaterium KM: thymine ${ }^{-}$methionine ${ }^{-}\left(\mathrm{KM}: \mathrm{T}^{-} \mathbf{M}^{-}\right)$has been described previously (4). E. coli WWU, a polyauxotrophic derivative of $E$. coli $15 \mathrm{~T}^{-}$with a block in the methionine biosynthetic pathway (1), was obtained from R. C. Bockrath of Indiana University, Indianapolis. Both organisms were grown in a basal medium consisting of $1.0 \%$ glucose $0.65 \%$ sodium L-glutamate, $0.3 \% \mathrm{~K}_{2} \mathrm{HPO}_{4}, 0.1 \% \mathrm{KH}_{2} \mathrm{PO}_{4}$, $0.1 \% \mathrm{Na}_{2} \mathrm{SO}_{4}, 0.005 \% \mathrm{MgSO}_{4}, 3 \times 10^{-4} \mathrm{M}$ ferric citrate, 1 to $5 \times 10^{-4} \mathrm{M}$ thymine, and $5 \times 10^{-4} \mathrm{M}$ DL-methionine (final $p H$ 7.0). For the growth of $E$. coli WWU, the basal medium was supplemented with $0.038 \mathrm{M} \mathrm{NH}_{4} \mathrm{Cl}$, and uracil, L-arginine, L-proline, and L-tryptophan at a final concentration of $5 \times 10^{-4} \mathrm{M}$ each. Cells were grown at $37 \mathrm{C}$ with vigorous aeration. Turbidity was determined with a Klett-Summerson colorimeter (filter no. 64). In each experiment, exponentially growing cells were harvested by centrifugation at $0 \mathrm{C}$, washed twice with cold, unsupplemented basal medium (minus thymine and methionine), and finally resuspended at about one-tenth cell density in the appropriately supplemented basal medium.

For viable cell counts, suspensions were diluted in the basal medium, and $0.05-\mathrm{ml}$ samples were spread on the surface of plates containing tryptoseyeast extract agar (3) supplemented with $5 \times 10^{-4}$ $\mathrm{M}$ thymine and $5 \times 10^{-4} \mathrm{M}$ DL-methionine. Plates

\footnotetext{
${ }^{1}$ Research Career Development Awardee (5-K03-AI-05038) of the National Institutes of Health.
}

were incubated for $24 \mathrm{hr}$ at $37 \mathrm{C}$ prior to counting colonies.

Photographs were taken with a Bausch and Lomb binocular phase-contrast microscope equipped with a Polaroid camera attachment and a flat-field oil immersion objective lens (100X; 1.25 N.A.). A droplet of cell suspension was placed on a slide containing a thin layer of $1 \%$ Ionagar no. 2 (Consolidated Laboratories, Inc., Chicago Heights, Ill.), covered with a cover slip, and photographed with Polaroid black and white, 300 speed/type 107 film.

All compounds were commercial samples of analytical grade. DL-Selenomethionine was obtained from K \& K Laboratories, Inc., Plainview, N.Y.

Figure 1 shows the comparative abilities of Se-M to substitute for S-M in methionineless strains of $E$. coli and $B$. megaterium. It can be seen that at equivalent concentrations, the maximum viable count achieved with Se-M is considerably less than that with S-M. Also, in general, S-M supports a more rapid rate of growth than Se-M. In several experiments with Se-M, maximum cell densities of $E$. coli were obtained with $10^{-4} \mathrm{M}$ Se-M, whereas with $B$. megaterium $5 \times$ $10^{-5} \mathrm{M}$ Se-M was optimal. As shown in Figure 1B, the viable count of $B$. megaterium increases approximately fourfold over a 2-hr period of amino acid starvation and then reaches a plateau. This is in contrast to the situation with $E$. coli WWU (Fig. 1A), and could be explained by the presence of a substantial intracellular methionine pool or by the fragmentation of cell chains. When $E$. coli WWU (starting with approximately $10^{8}$ viable cells $/ \mathrm{ml}$ ) was subcultured in media containing $10^{-4} \mathrm{M}$ Se-M in place of S-M, there was a progressive decrease in the growth rate, with a cessation of growth after about five doublings. Continued incubation resulted in the selection of methionine $^{+}$revertants. In a similar experiment 

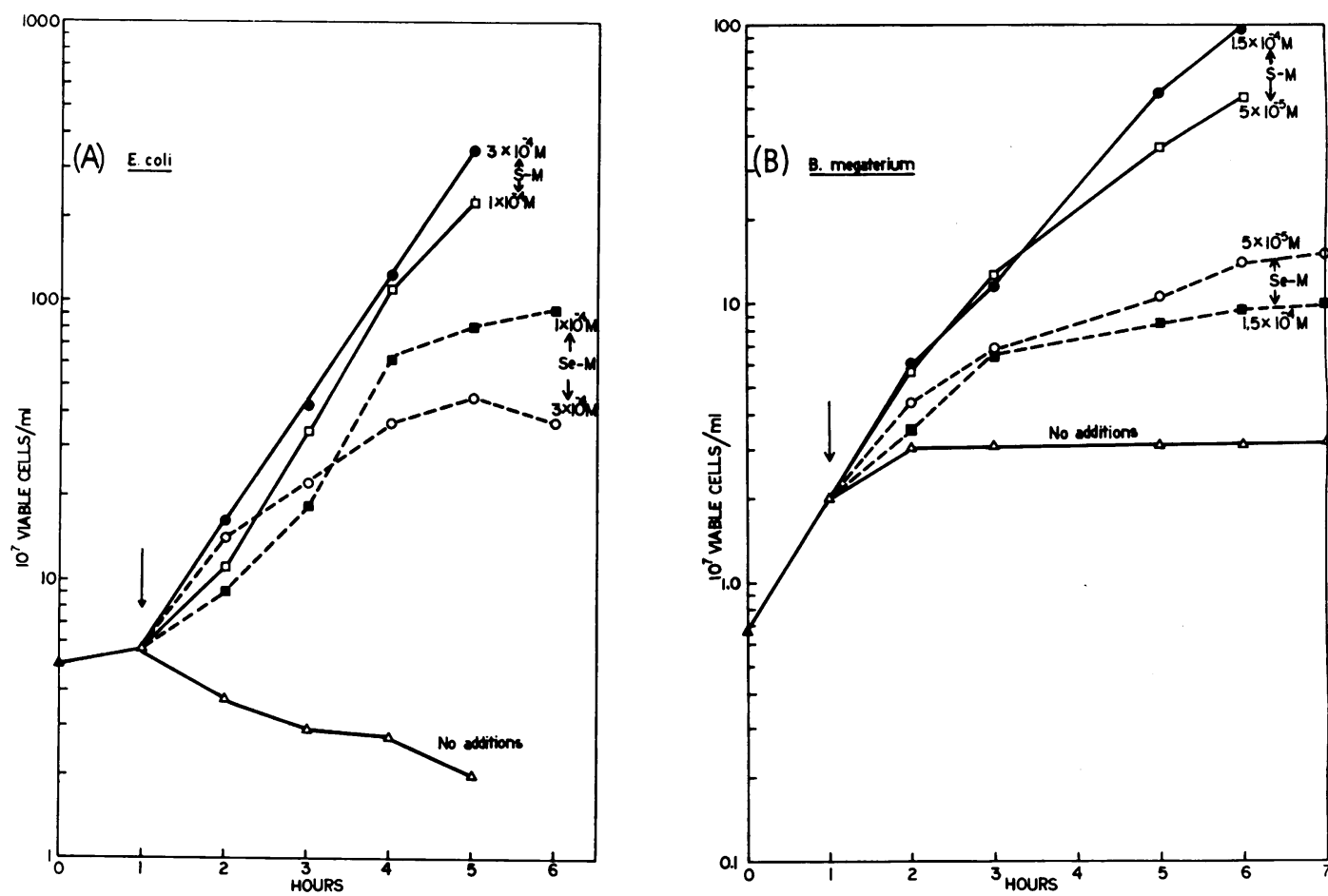

FIG. 1. Comparative effects of DL-methionine $(S-M)$ and $D L$-selenomethionine $(S e-M)$ on viability of $(A) E$. coli and $(B) B$. megaterium. Methionine-grown cells were harvested and washed as described. Cells were suspended in the complete basal medium in the absence of methionine and were shaken for $1 \mathrm{hr}$ at $37 \mathrm{C}$ to deplete endogenous methionine pools. Additions were made at the times indicated by the arrow.

with $B$. megaterium (starting with approximately $10^{7}$ to $2 \times 10^{7}$ viable cells $/ \mathrm{ml}$ ), growth ceased after about three doublings in the presence of $5 \times 10^{-5} \mathrm{M}$ Se-M. This is in contrast to a methionineless strain of $E$. coli ML-30 which has been reported to grow exponentially through more than 100 generations in a minimal liquid containing $10^{-4} \mathrm{Se}-\mathrm{M}$ in place of S-M (2); the generation time with Se-M was found to be about $30 \%$ greater than that with S-M. Thus, both $E$. coli WWU and $B$. megaterium KM differ from $E$. coli ML-30 in that they can use Se-M in place of S-M for only a very limited number of doublings.

The ability of Se-M to support thymineless death is shown in Fig. 2. It can be seen that for the first $2 \mathrm{hr}$ both Se-M and S-M support similar rates of thymineless death in $E$. coli (Fig. 2A). With B. megaterium, on the other hand (Fig. 2B), Se-M is a poor substitute for S-M. Notice that double starvation (minus thymine and methionine) is not lethal for $B$. megaterium but is lethal for $E$. coli.

When $E$. coli WWU is grown with $10^{-4} \mathrm{M}$ Se-M instead of S-M for about five doublings, the normally rod-shaped cells are converted to spherical or oval forms (Fig. 3). Exposure of $B$. megaterium to $5 \times 10^{-5} \mathrm{M}$ Se-M does not result in any morphological change. In an experiment involving the preparation of cell extracts from $E$. coli WWU, it was found that although the rod-shaped cells are lysed by exposure to lysozyme-Versene, the spherical or oval cells are resistant to lysis.

FIG. 2. Comparative abilities of DL-methionine $(S-M)$ and $D L$-selenomethionine (Se-M) to support thymineless death in $(A) E$. coli and $(B) B$. megaterium. Methionine-grown cells were harvested and washed as described. Cells were suspended in the complete basal medium in the absence of thymine and methionine and the above additions were made at zero time. E. coli $=1 \times 10^{-4}$ M S-M or Se-M. B. megaterium $=5 \times 10^{-5}$ M S-M or Se-M. Incubation was at $37 \mathrm{C}$.

FIG. 3. Morphology of $E$. coli WWU grown in basal medium containing $(A) I \times 10^{-4} M$ DL-methionine and $(B) 1 \times 10^{-4} M$ DL-selenomethionine. Final magnification, approximately 1,800X. 

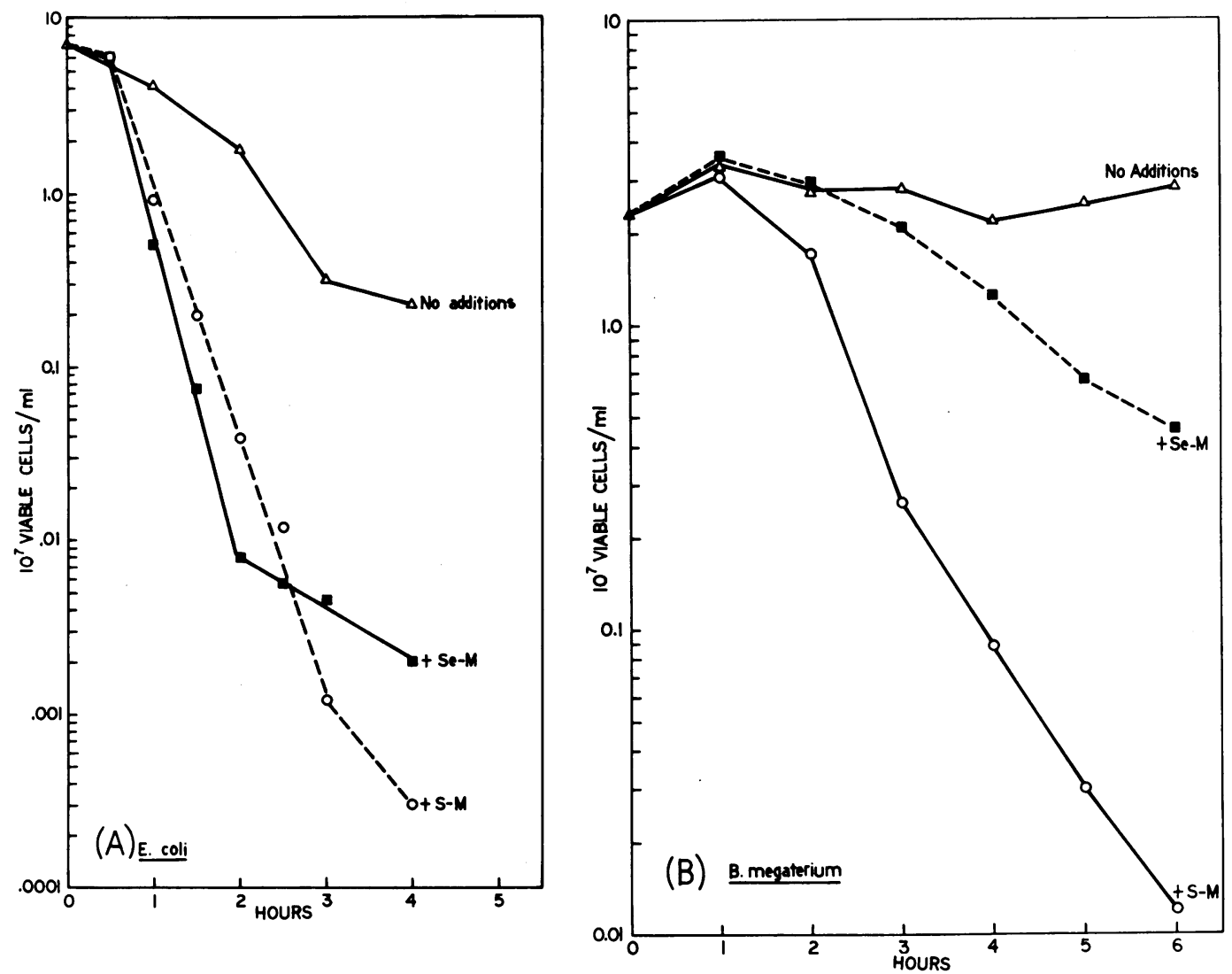

FIG. 2

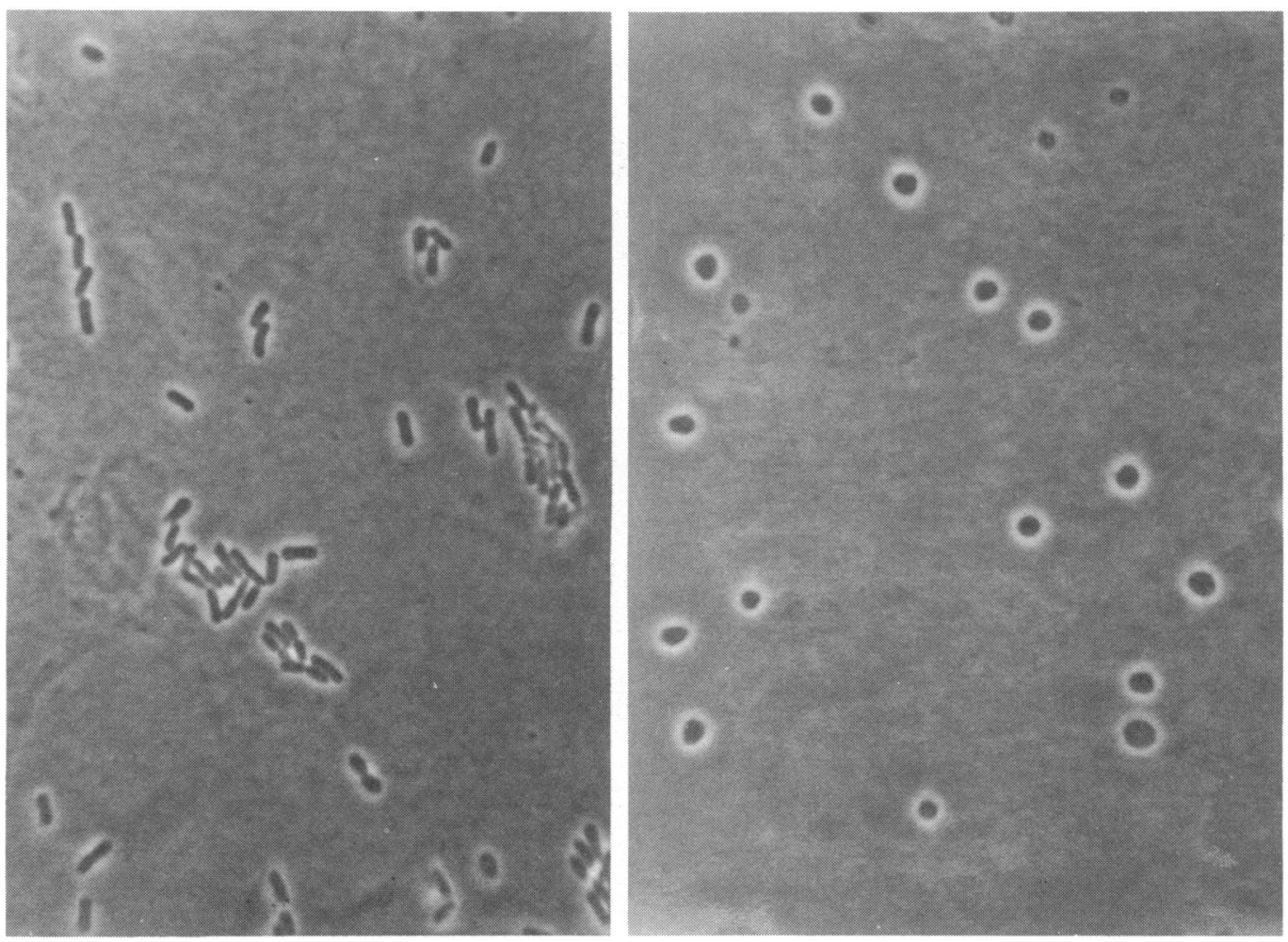

Fig. 3 
This investigation was supported by Public Health Service grant HD-02566 from the National Institute of Child Health and Human Development.

\section{LITERATURE GITED}

1. Bockrath, R. C., M. Osborn, and S. Person. 1968. Nonsense suppression in multiauxotrophic derivatives of Escherichia coli $15 \mathrm{~T}^{-}$: identification and consequences of an amber triplet in the deoxyribomutase gene. J. Bacteriol. $9: 146-153$.
2. Cowie, D., and G. Cohen. 1957. Biosynthesis by Escherichia coli of active altered proteins containing selenium instead of sulfur. Biochim. Biophys. Acta 26:252-261.

3. Friedman, M., and P. B. Cowles. 1953. The bacteriophages of Bacillus megaterium. I. Serological, physical, and biological properties. J. Bacteriol. 66:379-385.

4. Wachsman, J. T., and V. Irwin. 1970. Methylated bases of Bacillus megaterium KM nucleic acids: a comparison with Escherichia coli. J. Bacteriol. 104:814-818. 